\title{
THE FORCE CONE METHOD: A NEW THINKING TOOL FOR LIGHTWEIGHT STRUCTURES
}

\author{
C. MATTHECK \& S. HALLER \\ Karlsruhe Institute of Technology (KIT), Germany.
}

\begin{abstract}
The force cone method developed by Claus Mattheck enables computer-free topology designing and offers a profound knowledge for lightweight structures. Thus, the recently developed method enhances the series of the so-called thinking tools. The method's basic idea is the force distribution of a single force in an elastic plane. The symmetrically placed cones appear in front of the force and behind it. These cones intersect with $90^{\circ}$ angles at primary points that quickly lead to a structural design proposal. Furthermore, the method is very useful for the evaluation of structures and their lightweight potential. With the knowledge of the load case, it is easy to identify the main tension and compression paths leading to a deeper understanding of lightweight results. Natural structures such as trees can also be understood in another way, highlighting the structural principles at the root, leaf, treetop or even the entire tree. Nowadays, technical lightweight solutions can be found with different methods, including the soft kill option developed at the KIT 20 years ago. The method resembles that of the biological mineralization process of living bone and results in structures that can be seen as optimized lightweight design proposals. The comparisons of those structures with the state-of-the-art designs used in the industry and with those found by the force cone method indicate the high potential of the new method. For the confirmation of the basic rules and principles, different assembly positions of force and supports as well as different types of supports, such as fixed supports or torsion anchors, have been analyzed.

Keywords: Force cone method, lightweight structure, topology design.
\end{abstract}

\section{INTRODUCTION}

In mechanical engineering, a design needs to meet several demands. At first, the design needs to withstand the given tasks with regard to applied loads and the durability during its lifetime. In addition to optical issues, nowadays the weight often counts the most. With the increasing energy costs, the weight reduction within mechanical constructions gets more and more importance. Thus, almost everything has to be lightweight.

In nature there are further reasons for lightweight designs. Running faster than someone makes hunting more effective or prevents from being eaten. Low weight enables flying and consequently unlocks new territory. Million years of evolution brought up an advanced degree of lightweight optimization. Several starting points exist, e.g. the skeleton as the structural framework of many species. The framework is built up of bones, muscles and sinews, resembling the first step of the lightweight design. The second step can be found with a closer look at the bones. The bone material is placed perfectly in correlation with the force flow. The highly loaded zones are filled with a trabecular bone, also called spongiform bone, which is a micro-framework of very fine small struts of the bone.

With the soft kill option (SKO), the lightweight principle of the bone growth was transferred into technical application and is used for the optimization of technical components. The optimized topology designs can be derived using that computer method. The force cone method enlarges the SKO. The method conveys an understanding of lightweight design. Without the use of a computer, optimized topology designs can be found and the given structures, both natural and man-made, can be understood and evaluated concerning the lightweight potential. 


\section{MATERIALS AND METHODS}

\subsection{Soft kill option}

The SKO was developed at the Karlsruhe Institute of Technology 20 years ago and it is one of the empirical topology optimization methods. With the given design spaces and the load and support boundary conditions, the method finds optimized topology designs, which can be used as design proposals [1].

The basic principle of the method was found in nature. The biological mineralization process of living bone is used as a model. Bone building also occurs as stress-controlled. In areas of higher stresses, bone-building cells called osteoblasts make sure that more material is accumulated. Where the stress is lower osteoclasts shrink away the weakly loaded or even unused material. The fins of the lamella structure shown in Fig. 1A are oriented in the main stress directions. This framework structure instead of the solid material reduces the weight [1].

The first step of the SKO procedure includes the determination of loads, supports and the maximum available design space (see Fig. 1B). After computing and analyzing the stress distribution, the material is made softer in places of lower stress. On the contrary, the young's modulus is raised in areas of higher stresses. With the new material properties, a new stress distribution can be computed. This is an iterative process and will be repeated until the separation of the weak and the strong material is sharp. The result depends on several parameters, e.g. the reference stress or the number of iterations, and it can look like the structure shown in Fig. 1C. This is a design proposal that still needs to be dimensioned [2].

\subsection{The force cone method}

The force cone method is one of the so-called thinking tools. Similar to all the thinking tools, the force cone method can be used without a computer. The method determines the topology designs and helps in understanding and evaluating the lightweight potential of a given structure.
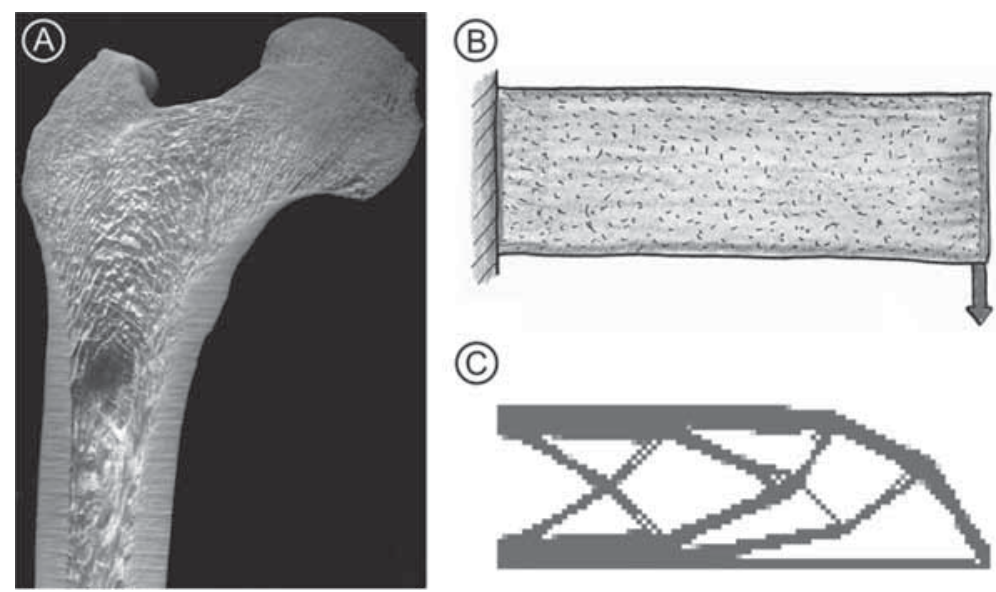

Figure 1: (A) The human bone (femur), (B) the maximum design space for the soft kill option (SKO) and (C) the lightweight design by the SKO [2]. 
The basic idea is that a single force in a huge elastic plane pushes a $90^{\circ}$ compression cone in front and pulls a $90^{\circ}$ tension cone behind. The $90^{\circ}$ angle becomes plausible when drawing the shear squares, which is another thinking tool (see Fig. 2A). The resulting compression and tension directions restrict the effective space of the cones.

This idea can be comprehended with the stress distribution of a single force in an elastic plane (analytic solution from [3]). Around $80 \%$ of the occurring radial stress will be included by a $90^{\circ}$ cone symmetrically placed in front of the force and behind it. The radial stress distribution is shown in Fig. 2B [2].

The approach of the force cone method is shown in Fig. 3. First, the boundary conditions need to be clarified. The direction and the point of application as well as the quantity, the arrangement and the type of supports (Fig. 3A) are important. The force cones of the loads are added, i.e. a tension cone behind the force and a compression cone in front of the force (Fig. 3B). The reaction forces arrange for additional force cones (Fig. 3C). The above-mentioned $90^{\circ}$ angle provides the perpendicular crossings of cone borders that are used as primary points (Fig. 3D). This is valid for orthogonal intersections of the tension and compression cone borders. In the next step, the primary points need to be connected (Fig. 3E) to obtain the finished lightweight structure (Fig. 3F). Then the compression
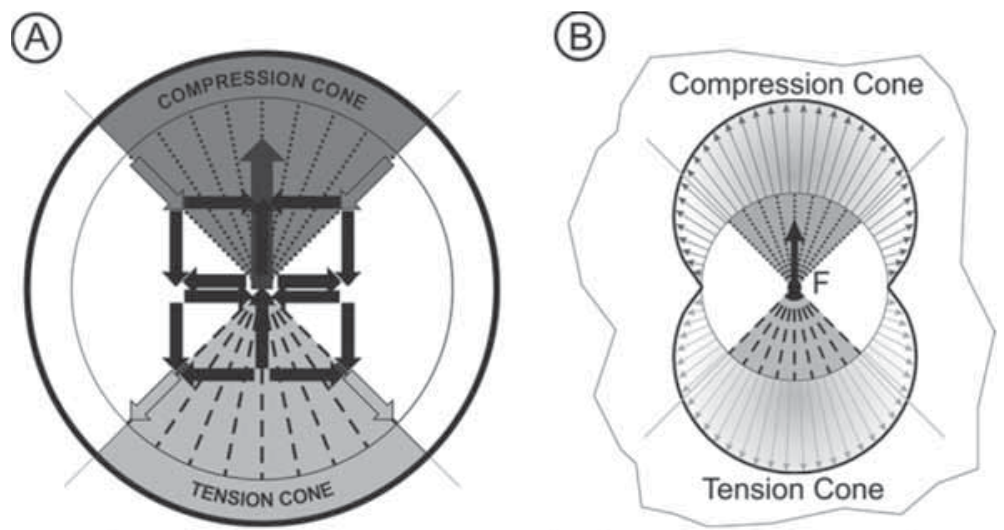

Figure 2: (A) The force cones constructed with the shear squares and (B) the radial stress distribution of a single force in an elastic plane [2].

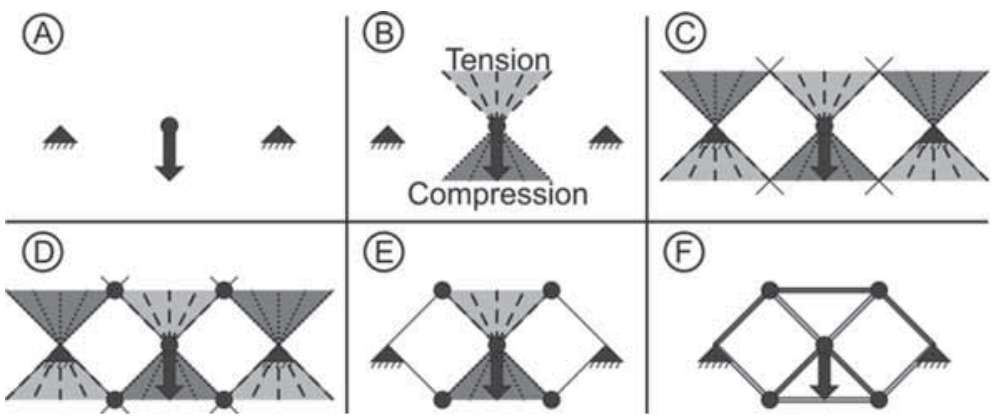

Figure 3: The stepwise approach of the force cone method [2]. 
struts (dark gray color) and tension ropes (bright gray color) can be easily determined. The topology design is a proposal that requires further dimensioning [2].

For a support in the shape of a torsion anchor, the force cone method provides another approach (see Fig. 4). Each point on the anchor circle can be seen as a primary point where compression and tension orthogonally intersect (Fig. 4A). The force will be looped around intersecting the tangents at right angles until the struts meet the anchor (Fig. 4B). For an infinite number of tangents, the result is the involute of the circle. The finished force cone structure is shown Fig. $4 \mathrm{C}$, and the SKO result is shown in Fig. 4D [2].

\section{RESULTS AND DISCUSSION}

Figures 5 and 6 show the transitions to obtuse angles. The obtuse angle is avoided in both the force cone method and the SKO, because of the higher stresses within the struts. Figure 5 shows a setup with a symmetrically placed force downwards between two fixed bearings. The force point of application is varied in height. As long as the struts lie within the force cone, the structure consists of only those direct struts. However, when the struts do not lie within the force cone, any more primary points lead to auxiliary constructions, i.e. the so-called gallows [4].

If the force points to the left, then the struts lie within the force cones, when the force point of application is closer to the supports. The auxiliary constructions are used for a higher point of application (see Fig. 6) [4].

(A)

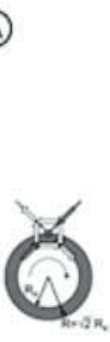

(B)

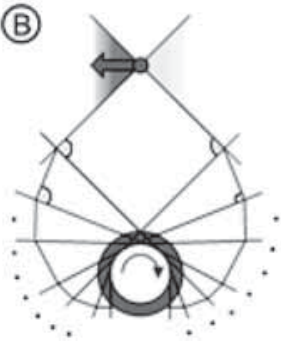

(C)

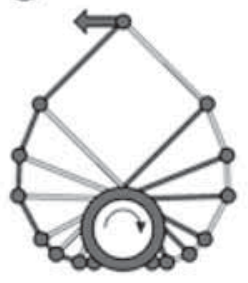

(D)

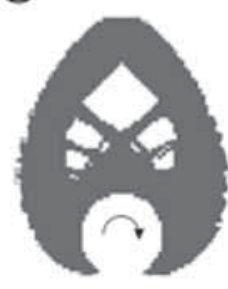

Figure 4: (A-C) The construction of a torsion anchor for central force introduction and (D) the SKO result for comparison [2].

(A)

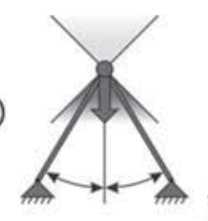

$\alpha<45^{\circ}$
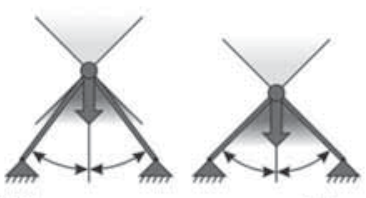

$\alpha_{\max }=45^{\circ}$

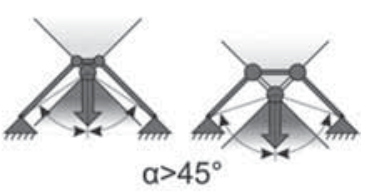

(B)
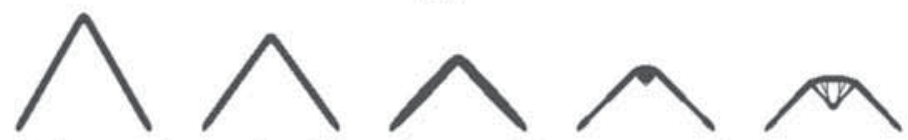

Figure 5: The symmetrically placed force downwards between two fixed supports: (A) the force cone method and (B) the SKO [4]. 
The setup of a force interacting with a torsion anchor was used to compare structures derived from the force cone method with state-of-the-art structures and the SKO structures (as shown in Fig. 7). For the comparison of these structures, the force was set constant and buckling of compression struts was added as a mode of failure. Thus, it is possible to compare the necessary materials to withstand the load without failure. As buckling is dependent on the magnitude of the force, the setup was calculated for different forces.

The calculated results are summarized in Table 1. Design A was used as the reference. The necessary material of other designs is relative to that number.

Design A as a wired beam is a construction that does not need a large number of connections but needs the most material. Design B with similarity to a ladder saves 19-30\% of the weight. Design C derived with the force cone method saves $\sim 16-54 \%$ of the weight. Design D generated with the SKO is even a little more better than the force cone method but needs resources such as computer, time and know-how.

Force cones can be found in nature and contribute to a better understanding of the results of the evolutionary optimization process. Figure 8 shows force cones within the tree. Rigid branches that are pressure resistant are acting as a series of compression cones and prevent the leaves from sliding down. The trunk would sink into the ground if there were no stiff roots forming the compression

(A)

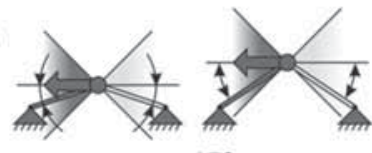

$\mathrm{a}<45^{\circ}$

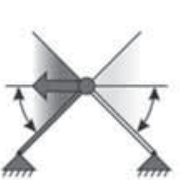

$\alpha_{\max }=45^{\circ}$

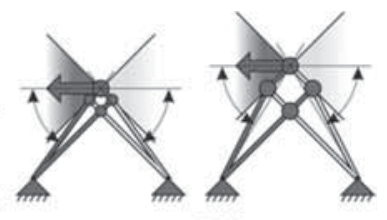

$\alpha>45^{\circ}$

(B)
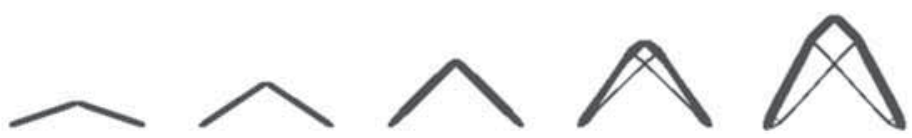

Figure 6: The symmetrically placed force to the left between two fixed supports: (A) the force cone method and (B) the SKO [4].

(A)

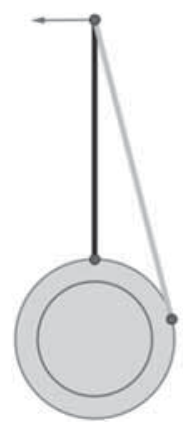

(B)

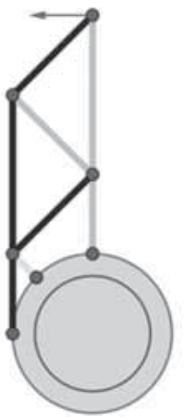

(C)

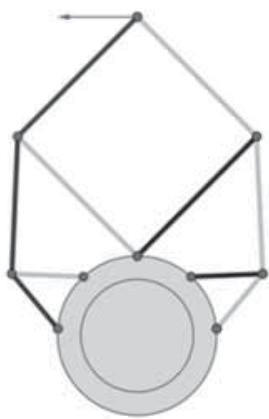

(D)

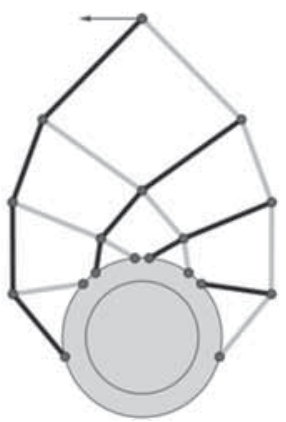

Figure 7: The structures for a torsion anchor as support: (A) a wired beam, (B) a ladder, (C) the force cone method with $42.5^{\circ}$ tangent angle and (D) the SKO. 
Table 1: The relative material for different designs of a torsion anchor support.

\begin{tabular}{lcccc}
\hline & Design A (\%) & Design B (\%) & Design C (\%) & Design D (\%) \\
\hline Small force & 100.0 & 81.7 & 84.4 & 78.0 \\
Middle force & 100.0 & 71.6 & 62.8 & 56.8 \\
High force & 100.0 & 70.4 & 46.6 & 43.2 \\
\hline
\end{tabular}
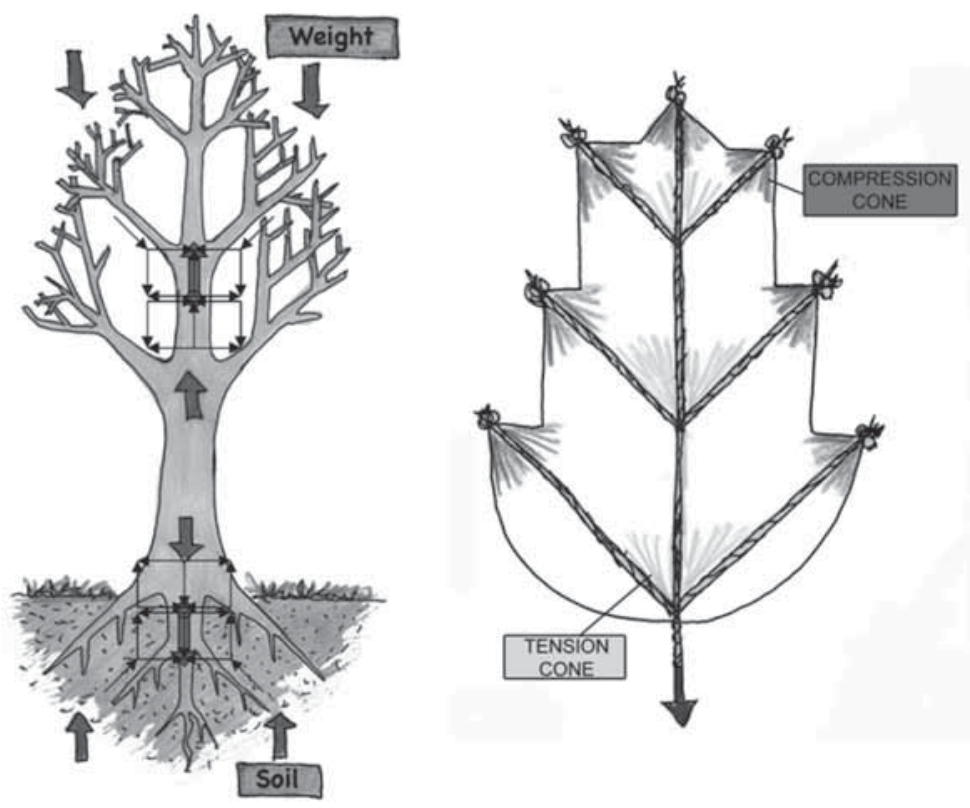

Figure 8: The force cones in nature [2].

cone pointing downwards. The leaves are exposed to wind. The main vein is collecting the lateral veins within a tension cone. The shape of the outermost contour line of the leaf can be reconstructed with compression cones at the end of the lateral veins [2].

\section{CONCLUSION}

The force cone method is a thinking tool that allows generating lightweight design proposals of the optimized quality. Comparisons with state-of-the-art structures show the huge potential of the easy-to-use method that seriously competes with computerized optimization methods but needs less resources. The method supports the understanding of lightweight structures, also the natural ones formed by the evolution.

\section{REFERENCES}

[1] Mattheck, C., Design in Nature, Springer-Verlag: Berlin and Heidelberg, 1998. doi: http:// dx.doi.org/10.1007/978-3-642-58747-4

[2] Mattheck, C., Thinking Tools after Nature, Verlag Karlsruher Institut für Technologie: Karlsruhe, 2011. 
[3] Kachanov, M., Shafiro, B. \& Tsukrov, I., Handbook of Elasticity Solutions, Kluwer Academic Publishers: Dordrecht, 2003.

[4] Mattheck, C., Haller, S. \& Bethge, K., Der verhasste stumpfe Winkel, Konstruktionspraxis 9, 2010.

This article is a reprint of the one presented at Design \& Nature VI Conference, 2012. 